\title{
THE RELATIONSHIP BETWEEN FRONTAL QRS-T ANGLE AND THE SEVERITY OF RECENTLY DIAGNOSED CHRONIC OBSTRUCTIVE PULMONARY DISEASE
}

\author{
${\text { Iclal Hocanl }{ }^{1} \text {, zulkif Tanrıverdi }}^{1}$, mehmet kabak ${ }^{2}$, fatıh gungoren ${ }^{1}$, and Mustafa Begenc \\ Tascanov $^{3}$ \\ ${ }^{1}$ Harran University Faculty of Medicine \\ ${ }^{2}$ mardin public hospital \\ ${ }^{3}$ Affiliation not available
}

April 10, 2021

\begin{abstract}
Background: Chronic Obstructive Pulmonary Disease (COPD) which is characterized by persistent airflow restriction and respiratory symptoms. Studies demonstrated that cardiac arrhythmias, cardiovascular mortality and cardiac death increased in these patients due to altered myocardial repolarization. Frontal QRS-T angle is a novel marker of myocardial depolarization and repolarization heterogeneity. In this study, we aimed to investigate the relationship between frontal QRS-T angle and COPD severity in patients with newly diagnosed COPD. Methods: A total of 104 newly diagnosed COPD patients without any significant comorbidities were included in this study. Patients were divided into two groups according to GOLD stage as follows: patients with mild and moderate COPD (group I) and severe and very severe COPD (group II). Frontal QRS-T angle was calculated from the automatic report of the electrocardiography device . Results: Frontal QRS-T angle was significantly higher in group II patients compared to in group I patients (43.0 [25.5-60.0] vs. 20.0 [12.0-32.0], P $<0.001)$. The best cut-off value of frontal QRS-T angle for predicting severe-very severe COPD was [?] 34.5 . Correlation analysis showed that frontal QRS-T angle was negatively correlated with FEV1/FVC $(\mathrm{r}=-0.524, \mathrm{P}<0.001)$ and MEF25-75 $(\mathrm{r}=-0.453, \mathrm{P}<0.001)$. In linear regression analysis, It was found that MEF25-75 ( $\beta$ : $-0.593, \mathrm{P}=0.006)$ was the only independent predictor of the frontal QRS-T angle. Conclusions: Frontal QRS-T angle, an easily obtainable marker form surface electrocardiography. In this study, we have shown for the first time that frontal QRS-T angle was significantly increased in patients with severe and very severe COPD.
\end{abstract}

THE RELATIONSHIP BETWEEN FRONTAL QRS-T ANGLE AND THE SEVERITY OF RECENTLY DIAGNOSED CHRONIC OBSTRUCTIVE PULMONARY DISEASE

Iclal Hocanli ${ }^{1}$, Zulkif Tanriverdi ${ }^{2} \mathrm{Mehmet} \mathrm{Kabak}^{3}$, Fatih Gungoren ${ }^{2}$, Mustafa Begenc Tascanov ${ }^{2}$

${ }^{1}$ Harran University, Faculty of Medicine, Department of Chest Diseases, Sanliurfa, Turkey

${ }^{2}$ Harran University, Faculty of Medicine, Department of Cardiology, Sanliurfa, Turkey

${ }^{3}$ Mardin Public Hospital, Clinic of Chest Diseases, Mardin, Turkey

Short title

QRS-T ANGLE AND OBSTRUCTIVE PULMONARY DISEASE

Corresponding author 
Iclal Hocanli,

Assistant Professor Doctor,

Mailing address: Harran University, Faculty of Medicine, Department of Chest Diseases, Sanliurfa, Turkey

Tlf: +904143444444

E-mail address: iclalhocanli2163@gmail.com

\title{
THE RELATIONSHIP BETWEEN FRONTAL QRS-T ANGLE AND THE SEVERITY OF RECENTLY DIAGNOSED CHRONIC OBSTRUCTIVE PULMONARY DISEASE
}

\begin{abstract}
Background: Chronic Obstructive Pulmonary Disease (COPD) which is characterized by persistent airflow restriction and respiratory symptoms. Studies demonstrated that cardiac arrhythmias, cardiovascular mortality and cardiac death increased in these patients due to altered myocardial repolarization. Frontal QRS-T angle is a novel marker of myocardial depolarization and repolarization heterogeneity. In this study, we aimed to investigate the relationship between frontal QRS-T angle and COPD severity in patients with newly diagnosed COPD.
\end{abstract}

Methods: A total of 104 newly diagnosed COPD patients without any significant comorbidities were included in this study. Patients were divided into two groups according to GOLD stage as follows: patients with mild and moderate COPD (group I) and severe and very severe COPD (group II). Frontal QRS-T angle was calculated from the automatic report of the electrocardiography device .

Results: Frontal QRS-T angle was significantly higher in group II patients compared to in group I patients (43.0 [25.5-60.0] vs. 20.0 [12.0-32.0], $\mathrm{P}<0.001$ ). The best cut-off value of frontal QRS-T angle for predicting severe-very severe COPD was [?] $34.5^{\circ}$. Correlation analysis showed that frontal QRS-T angle was negatively correlated with FEV1/FVC $(\mathrm{r}=-0.524, \mathrm{P}<0.001)$ and MEF25-75 $(\mathrm{r}=-0.453, \mathrm{P}<0.001)$. In linear regression analysis, It was found that MEF25-75 $(\beta$ : $-0.593, \mathrm{P}=0.006)$ was the only independent predictor of the frontal QRS-T angle.

Conclusions: Frontal QRS-T angle, an easily obtainable marker form surface electrocardiography. In this study, we have shown for the first time that frontal QRS-T angle was significantly increased in patients with severe and very severe COPD.

Keywords: Chronic Obstructive Pulmonary Disease; Disease severity; Myocardial repolarization; Frontal QRS-T angle.

\section{WHAT'S KNOWN?}

Frontal QRS-T angle, which is a novel marker of the heterogeneity in myocardial depolarization and repolarization, is described as the absolute difference between myocardial depolarization (QRS- axis) and repolarization ( $\mathrm{T}$ axis). It can easily be measured by using the 12-lead surface electrocardiography (ECG).The frontal QRS-T angle has been shown to be valuable in predicting many cardiac and non-cardiac diseases.

\section{WHAT'S NEW?}

Ours is the first research study that demonstrated an association between frontal QRS-T angle and severevery severe COPD. The primary finding of our study was that the patients with severe-very severe COPD had significantly higher frontal QRS-T angle than patients with mild-moderate COPD.It can be concluded that the patients who have an increased frontal QRS-T angle on initial ECG will have worse pulmonary function test results than the patients who do not have.

\section{INTRODUCTION}


Chronic Obstructive Pulmonary Disease (COPD), which is characterized by persistent airflow restriction and respiratory symptoms, is one of the most common causes of death globally ${ }^{1}$. Previous studies have demonstrated that COPD patients are at increased risk of cardiovascular morbidity and mortality ${ }^{2-4}$. Cardiac arrhythmias and sudden cardiac death (SCD) are very common in these patients ${ }^{3,5}$. The underlying mechanism of the relationships among COPD, cardiac arrhythmias, and SCD has not been clearly elucidated yet. Nevertheless, hypoxemia, autonomic dysfunction and acid-base disturbances can causes the improving of cardiac arrhythmias and SCD by altering myocardial repolarization in these patients ${ }^{6}$.

Alteration in myocardial repolarization has a crucial role in improving cardiac arrhythmias and SCD 7,8 . Frontal QRS-T angle, which is a novel marker of the heterogeneity in myocardial depolarization and repolarization, is described as the absolute difference between myocardial depolarization (QRS- axis) and repolarization ( $\mathrm{T}$ axis) ${ }^{9}$. It can easily be measured by using the 12-lead surface electrocardiography (ECG), and its abnormality reflects the electrical instability of the myocardium ${ }^{10}$. The frontal QRS-T angle has been shown to be valuable in predicting many cardiac and non-cardiac diseases ${ }^{11}$.

To our knowledge, there is no research that investigated the relationship between the frontal QRS-T angle and the severity of COPD. Our objective in this study is to evaluate the relationship between the frontal QRS-T angle and the severity of recently diagnosed COPD.

\section{METHODS}

The patients, who were recently diagnosed with COPD in the Chest Diseases Outpatient Clinics of the Harran University Faculty of Medicine, were enrolled in the study. The exclusion criteria consisted of the presence of diabetes mellitus, hypertension, chronic or acute heart failure, moderate or severe valvular heart disease, hyperthyroidism, anemia, sleep disorders, ECG signs of incomplete or complete bundle branch block. The local ethics committee approved the study, which was conducted in full compliance with the Declaration of Helsinki. The signed informed consent were obtained from all patients.

Tests for Pulmonary Function and Diffusing Capacity of the Lung Carbon Monoxide (DLCO) as recommended by the American Thoracic Society (ATS) were performed in all patients ${ }^{12}$. The assessment of pulmonary function was performed with at least three forced expiratory maneuvers in $90^{\circ}$ upright position with the nose closed, and the best value was recorded. The measurements of Forced Vital Capacity (FVC), Forced Expiratory Volume in 1 second (FEV1), and the ratio of FEV1 / FVC were recorded. The current guideline was used for COPD diagnosing and staging [1]. Accordingly, a post-bronchodilator FEV1 / FVC < $70 \%$ in the presence of clinical findings indicates persistent airflow limitation and is essential for diagnosing COPD. The GOLD (Global Obstructive Lung Disease ) stage of COPD is categorized as stage 1 (mild) when post-bronchodilator predicted FEV1 is equal to or greater than $80 \%$, stage 2 (moderate) when between $50 \%$ and less than $80 \%$, stage 3 (severe) when between $30 \%$ and less than $50 \%$, and stage 4 (very severe) when less than $30 \%$. Accordingly, the current study population was categorized into two groups as Group I, including the mild and moderate COPD patients, and Group II, including the severe and very severe COPD patients. The single-breath method via the Morgan Benchmark transfer test was used for the quantification of DLCO.

A 12-lead basal ECG with a $0.16-100 \mathrm{~Hz}$ filter range, $25 \mathrm{~mm} / \mathrm{s}$ speed, and $10 \mathrm{~mm} / \mathrm{mV}$ height both at rest and in the supine position was obtained from all patients on admission. The automatically reported axes of QRS and T wave were used to calculate the frontal QRS-T angle by using the formula: Frontal QRS-T angle $=-\mathrm{QRS}$ axis $-\mathrm{T}$ axis - . When the angle was greater than $180^{\circ}$, its subtraction from $360^{\circ}$ was used for the calculation, as previously reported ${ }^{13}$. Figure 1 depicts an example of the frontal QRS-T angle calculated as explained.

Blood samples for laboratory tests, including complete blood count and biochemical parameters consisting of serum electrolytes, liver enzymes, urea, and creatinine, were obtained from all patients on admission.

\section{Statistical Analysis}

SPSS for Windows version 21.0 (SPSS Inc., IL, USA) was used for statistical analyses. Kolmogorov-Smirnov test was used for evaluating if the continuous data were distributed normally. Continuous data that were 
expressed as mean +- SD or median (25-75 IQR) were compared using the Student $t$ or Mann-Whitney U tests, while categorical data that were expressed as the number and the percentage were compared using the Chi-square test. The correlation analysis was performed by using the Pearson or Spearman correlation coefficients. In order to predict the severity of COPD by using the frontal QRS-T angle, the cut-off value was determined using the receiver operating characteristics (ROC) curve analysis. The independent predictors of frontal QRS-T angle were determined using the linear regression analysis. Value of $p<0.05$ was considered statistically significant.

\section{RESULTS}

A total of 104 COPD patients without any significant comorbidities were included in this study. Mild and moderate COPD (group I) was detected in 35 patients, whereas severe and very severe COPD (group II) was detected in 69 patients according to the COPD GOLD stage. Comparison of baseline characteristics and laboratory parameters between two groups are listed in Table 1. No significant difference was found between two groups.

Spirometry and pulmonary function tests are demonstrated in Table 2. Patients in group II had significantly lower FVC $(2.9$ [2.0-3.6] vs. 1.8 [1.4-2.2], $\mathrm{P}<0.001)$, FEV1 (1.9 [1.3-2.3] vs. $1.0[0.7-1.3], \mathrm{P}<0.001]$, FEV1/FVC (67.0 [62.0-69.0] vs. 55.0 [49.5-64.0], $\mathrm{P}<0.001)$, MEF25-75 (1.1 [0.8-1.4] vs. 0.6 [0.4-0.9], $\mathrm{P}<$ $0.001)$ and TLC (5.6 [4.3-6.2] vs. $4.7[3.7-5.2], \mathrm{P}=0.003)$ values compared to patients in group I.

Comparison of frontal QRS-T angle between two groups is shown in Figure 2. Frontal QRS-T angle was significantly higher in group II patients than in group I patients $(43.0$ [25.5-60.0] vs. 20.0 [12.0-32.0], P < 0.001). ROC curve analysis was performed to determine the best cutoff value of frontal QRS-T angle for predicting severe-very severe COPD (Figure 3). Frontal QRS-T angle [?] 34.5deg predicted severe-very severe COPD with a sensitivity of $63.8 \%$ and specificity of $89.2 \%$ (AUC: $0.752, \mathrm{P}<0.001,95 \%$ CI: $0.657-0.848$ ).

Correlation analysis of frontal QRS-T angle with other variables is shown in Figure 4. Frontal QRS-T angle was negatively correlated with FEV1/FVC $(\mathrm{r}=-0.524, \mathrm{P}<0.001)$ and MEF 25-75 ( $\mathrm{r}=-0.453, \mathrm{P}<0.001)$.

Multivariable logistic regression analysis demonstrated that frontal QRS-T angle was the independent predictor of severe-very severe COPD (odds ratio: 1.051, 95\% confidence interval: $1.024-1.079, \mathrm{P}<0.001$ ) (Table 3). Linear regression analysis was used to determine the independent predictors of frontal QRS-T angle. It was found that MEF25-75 ( $\beta$ : $-0.593, \mathrm{P}=0.006)$ was the only independent predictor of the frontal QRS-T angle (Table 4).

\section{DISCUSSION}

In the current study, we researched the significance of frontal QRS-T angle in recently diagnosed COPD patients. The primary finding of our study was that the patients with severe-very severe COPD had significantly higher frontal QRS-T angle than patients with mild-moderate COPD. To the best of our knowledge, ours is the first research study that demonstrated an association between frontal QRS-T angle and severe-very severe COPD.

COPD is a disease in which there is an irreversible limitation of airflow and an increased inflammatory response in the lungs. Hypoxia, which is the main finding of the disease, gets worse as the disease progresses ${ }^{14}$. The hypoxia contributes to the deterioration of the quality of life, increased morbidity, disability, and mortality ${ }^{15}$. Moreover, the pathologic changes in COPD can alter the cardiac repolarization and depolarization. Thus, the increased risk of mortality due to cardiovascular causes in the patients has been principally attributed to cardiac arrhythmias and SCD due to the altered cardiac repolarization and depolarization ${ }^{2-5,16}$. Although the main mechanism of the altered cardiac repolarization and depolarization in the patients with COPD has not been clearly elucidated, it has been suggested that hypoxemia, autonomic dysfunction, and acid-base disturbances might be the potential causes ${ }^{6,8,17}$.

Alteration in myocardial repolarization represents an increased risk for malign cardiac arrhythmia and SCD 7,8. Myocardial repolarization has been traditionally evaluated considering the QT interval on surface ECG 
18. However, the calculation of QT interval is not easy and requires devices like ruler and magnifying glass, as well as sophisticated software programs. Besides, the reproducibility of QT interval measurements is low ${ }^{19}$. For this reason, most investigators have focused research studies on identifying the novel ECG parameters that can be easier to calculate using the surface ECG. Frontal QRS-T angle may be used as a basic marker that reflects the absolute difference between the repolarization and depolarization of the myocardium ${ }^{9,10}$. Investigations have shown that frontal QRS-T angle was less sensitive to sound than the measurements of QT interval ${ }^{13,20}$. Moreover, the calculation of the frontal QRS-T angle did not require any additional tools and was much easier than calculating the QT interval. Furthermore, it has been shown that the increase in the frontal QRS-T angle was an earlier event than the development of overt electrocardiographic changes 11. The axes of QRS and T are automatically reported by the vast majority of ECG devices; therefore, the calculation of the frontal QRS-T angle can be readily performed incorporating those measurements in the formula Frontal QRS-T angle $=-$ QRS axis $-\mathrm{T}$ axis-.

The clinical importance of QT interval in the patients with COPD had been investigated in previous studies. It was found that the QTc interval and QT dispersion were significantly longer in COPD patients when compared to controls ${ }^{8,21}$. Zulli et al. demonstrated that the increased QT dispersion was an important independent predictor of pulmonary and cardiovascular mortality in COPD patients ${ }^{22}$. Moreover, it was reported that the increased QT dispersion could decrease after the partial correction of the hypoxemia by medical treatment. Those results suggested that the variations in blood gas levels might be critical for the increased QT interval in the patients with COPD ${ }^{23}$. Although QT interval was comprehensively evaluated in COPD, there are no studies that investigated the importance of frontal QRS-T angle in the recently diagnosed COPD patients.

We studied for the first time that the patients with severe and very severe COPD had significantly increased frontal QRS-T angle compared to the patients with mild and moderate COPD. Additionally, significant reductions in the ratio of FEV1/FVC and the levels of MEF25-75 were detected in severe and very severe COPD patients. We observed that the predictive value of a frontal QRS-T angle[?] $30^{\circ}$ for severe to very severe COPD had a sensitivity and a specificity of $73 \%$ and $67 \%$, respectively, while the ratio of FEV1/FVC was found to be an independent predictor of the frontal QRS-T angle. Our findings suggest that the frontal QRS-T angle has a very important clinical role in patients with COPD. It can be concluded that the patients who have an increased frontal QRS-T angle on initial ECG will have worse pulmonary function test results than the patients who do not have.

Previous studies showed that the frontal QRS-T angle could be wider in the patients with various conditions like hypertension and diabetes mellitus ${ }^{20,24,25}$. To exclude the possible effects of comorbid factors in elucidating the relationship between frontal QRS-T angle and COPD, we included only the COPD patients without any comorbidities. Therefore, the number of patients in our study remained relatively small, which could be considered as a limitation of the study. In addition, drugs that are used in the treatment of COPD may change the myocardial repolarization. So, to exclude the possible effect of COPD drugs on frontal QRS-T angle, we evaluated the patients recently diagnosed with COPD and who had not been started on a medication regimen yet. We consider that the choice of inclusion criteria had strengthened our study. Some other limitations of our study should also be acknowledged. As mentioned earlier, the small size of the study group had been further limited by the lack of a control group that did not permit us to investigate our results in a comparative way. Second, the patients were not followed up for any possible cardiovascular complications. It would be better to use a 24-hour Holter ECG for that purpose, and the associations among frontal QRS-T angle, arrhythmias, and SCD could be demonstrated more clearly.

In conclusion, based on the current study results that indicated a significant increase in the frontal QRS-T angle in severe to very severe COPD patients, we suggest that frontal QRS-T angle can be used as a novel electrocardiographic marker which can be readily calculated using the surface ECG. Nevertheless, further prospective studies with larger population sizes are needed to verify our results and to demonstrate the relationship between the frontal QRS-T angle and COPD.

\section{Hosted file}


copd-ekg figures.pdf available at https://authorea.com/users/407010/articles/517455-therelationship-between-frontal-qrs-t-angle-and-the-severity-of-recently-diagnosed-chronicobstructive-pulmonary-disease

\section{Hosted file}

copd-ekg, TABLES.pdf available at https://authorea.com/users/407010/articles/517455-therelationship-between-frontal-qrs-t-angle-and-the-severity-of-recently-diagnosed-chronicobstructive-pulmonary-disease 